\title{
Persistent ERK activation maintains learning-induced long-lasting modulation of synaptic connectivity
}

\author{
Sivan Ida Cohen-Matsliah, Yaron Seroussi, Kobi Rosenblum, and Edi Barkai ${ }^{1}$ \\ Departments of Neurobiology and Biology, Faculty of Sciences, Haifa University, Haifa 31905, Israel
}

\begin{abstract}
Pyramidal neurons in the piriform cortex from olfactory-discrimination (OD) trained rats undergo synaptic modifications that last for days after learning. A particularly intriguing modification is reduced paired-pulse facilitation (PPF) in the synapses interconnecting these cells; a phenomenon thought to reflect enhanced synaptic release. The molecular machinery underlying this prolonged physiological modulation of synaptic connectivity is yet to be described. We have recently shown that extracellular regulated kinase (ERK) pathway and protein kinase $C$ (PKC) are also required for learning-induced enhancement of intrinsic neuronal excitability. Here we examine whether these signal-transduction cascades are instrumental for the learning-induced, long-lasting PPF reduction. Days after learning completion, PD98059, a selective inhibitor of MEK, the upstream kinase of ERK, increased PPF in neurons from trained, but not in neurons from naïve and pseudo-trained rats. Consequently, the differences in PPF between neurons from trained rats and controls were abolished. The level of activated ERK in synaptoneurosomes was significantly higher in piriform cortex samples prepared from trained rats. Notably, ERK activation revealed that PPF reduction lags behind ERK activation by 2 d. Similarly, the PKC blocker, GF-109203X, enhanced PPF in neurons from trained rats only, thus abolishing the differences between groups. Interestingly, the PKC activator, OAG, had no effect, indicating that PKC activation is required, but not sufficient for long-lasting PPF reduction. Our data show that persistent ERK activation has a key role in maintaining learning-induced PPF reduction for days. This time frame of compartmental ERK-dependent synaptic modulation suggests a novel role for ERK in cortical function.
\end{abstract}

Learning of a particularly difficult olfactory-discrimination (OD) task results in acquisition of rule learning. Such rule learning is manifested in a dramatic enhancement of learning capability; while learning to discriminate between the first pair of odors required 7-8 d of training, discrimination between subsequent pairs of odors is achieved almost immediately (Saar et al. 1998, 1999). This remarkable enhancement in learning capability, termed "rule learning," suggests that profound changes should occur in the relevant brain areas.

Indeed, OD rule learning is accompanied by a series of profound physiological and morphological modifications in synaptic transmission between layer II piriform cortex (PC) pyramidal neurons (Saar et al. 1999, 2002; Knafo et al. 2005; Brosh et al. 2007). A particularly interesting modification is a long-lasting reduction in paired-pulse facilitation (PPF) (Saar et al. 1999; Brosh et al. 2007). Such reduction occurs 3 d after training completion and lasts for $5 \mathrm{~d}$, and is thought to reflect enhanced synaptic release (Saar et al. 1999), which serves to maintain high learning capability, but not storage of memory for a specific odor. The mechanism underlying such long-lasting, presumably presynaptic, learning-induced modification is yet to be described.

A wide range of studies indicates the clear importance for extracellular signal-regulated kinase (ERK) in memory formation across many species and brain areas (for review, see Adams and Sweatt 2002). In a time frame of minutes and up to a few hours after acquisition of fear conditioning (Atkins et al. 1998; Schafe et al. 2000), taste learning (Berman et al. 1998; Belelovsky et al. 2005), classical conditioning (Crow et al. 1998), and water-maze training (Blum et al. 1999) ERK activation increases transiently.

The best-studied form of synaptic plasticity in the central nervous system is long-term potentiation (LTP) of excitatory synaptic transmission. LTP is an activity-dependent strengthening of

'Corresponding author.

E-mail ebarkai@research.haifa.ac.il; fax 972-8-8288108.

Article is online at http://www.learnmem.org/cgi/doi/10.1101//m.1127008. synaptic efficacy that has been proposed to be a cellular model for memory formation. The involvement of ERK in this form of plasticity is well established. ERK activation was found to be necessary for both induction and consolidation of LTP (English and Sweatt 1996, 1997; Rosenblum et al. 2000, 2002; Di Cristo et al. 2001; Selcher et al. 2003). Moreover, persistent ERK activation is required for long-term maintenance of learning-induced enhancement of intrinsic neuronal excitability (Cohen-Matsliah et al. 2007).

Several processes can underlie enhancement of synaptic transmission, such as LTP. Insertion of $\alpha$-amino-3-hydroxy-5methyl-4-isoxazole propionic acid receptors (AMPARs) into the synapse, regulation of potassium channels, dendritic spine enlargement, and appearance of new filopodia and spines are molecular and structural modifications that can underlie the increased synaptic transmission. All of these changes were shown to be dependent on ERK activation (for review, see Sweatt 2004; Thomas and Huganir 2004).

The purpose of the present study was to examine the role of ERK and its upstream kinase, PKC, in the maintenance of learning-induced reduction in PPF.

\section{Results}

Recordings in neurons from trained and pseudo-trained rats were performed $3 \mathrm{~d}$ after the last training session, when learningrelated reduction in paired-pulse facilitation (PPF) first appears (Saar et al. 1999). In control saline Ringer solution (NSR), the averaged PPF in neurons from trained rats was significantly smaller compared with the averaged PPF values in neurons from pseudo-trained and naïve rats (Fig. 1), confirming our earlier report (Saar et al. 1999). Resting membrane potential $(-79.3$ $\mathrm{mV} \pm 7.3, n=34$ in naïve, $-79.2 \pm 7.0, n=49$ in trained, and $-82.0 \pm 7.4, n=34$ in pseudo-trained) and input resistance (33.4 Mohms $\pm 12.7, n=24$ in naïve, $30.0 \pm 8.4, n=30$ in 
A
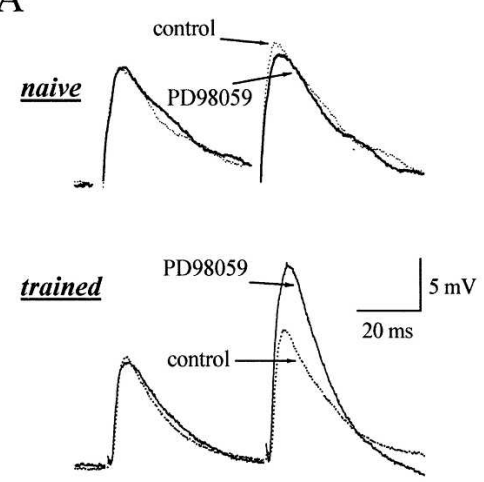

B

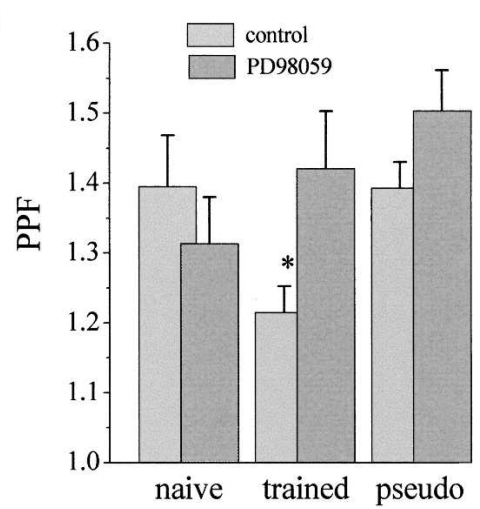

Figure 1. Learning-induced PPF reduction is dependent on ERK activation. $(A)$ PPF was measured in response to two stimuli, with an interstimulus interval of $50 \mathrm{msec}$. Neuron was recorded at membrane potential of $-80 \mathrm{mV}$. Each trace is a digital average of 10 responses to stimuli applied at $0.1 \mathrm{~Hz}$. While PD98059 has no effect on PPF on a neuron from a pseudo-trained animal (upper trace), it increases PPF in a neuron from a trained rat (bottom trace). (B) As previously reported (Saar et al. 1999), the mean PPF in neurons from trained rats was significantly smaller compared with that in neurons from naïve and pseudo-trained. PD98059 application enhanced PPF in the trained group only, as result of which the difference between the trained and the two control groups was abolished. PPF in NSR was measured in 18 neurons from nine naïve rats, 19 neurons from 11 trained rats, and 18 neurons from 11 pseudo-trained rats. PPF in PD98059 was measured in 18 neurons from seven naïve rats, nine neurons from five trained rats, and 11 neurons from five pseudo-trained rats. Values represent mean \pm SE.

trained, and $35.8 \pm 10.9, n=18$ in pseudo-trained) did not differ between neurons from the three groups.

\section{Learning-induced long-lasting PPF reduction is not maintained after ERK inhibition}

To examine whether the prolonged reduction in PPF is maintained by ERK activation, we applied the MEK-specific inhibitor PD98059 $(38 \mu \mathrm{M})$. PD98059 significantly increased the PPF value in neurons from trained rats only. In neurons from naïve and pseudo-trained rats the averaged PPF value was not affected by the inhibitor. Two typical examples of the different effect of PD98059 on neurons from a pseudo-trained and a trained rat are shown in Figure 1A. In neurons from trained rats the averaged PPF was significantly increased from $1.23 \pm 0.15(n=19)$ in NSR to $1.42 \pm 0.25(n=9)$ in PD98059 $(P<0.05)$. For naïves, the aver- aged values were $1.40 \pm 0.31,(n=18)$ in NSR and $1.31 \pm 0.28(n=18)$ in PD98059. For pseudo-trained, the averaged values were $1.38 \pm 0.15(n=18)$ in NSR and $1.50 \pm 0.19(n=11)$ in PD98059.

Although the MEK inhibitor seemed to have a tendency to affect neurons from the pseudo-trained group too, the difference in PPF before and after PD98059 application stop short of being significant $(P=0.1)$. Following PD98059, no differences in PPF were observed between neurons of learning and control rats (Fig. 1B). These results further suggest that ERK activation is necessary for the maintenance of learningrelevant enhancement in synaptic transmission when it first appears.

\section{Olfactory-discrimination learning induces long-term ERK activation in synaptoneurosomes}

Since learning-induced enhancement of synaptic transmission is dependent of persistent ERK activation, we hypothesized that learning should be accompanied by an increased ERK phosphorylation in the synapses. Using Western blot analysis, we measured the total amount of ERK and its phosphorylation state as a measure of activation in the three experimental groups. The total amount of ERKII (normalized to naïve) was similar between the groups $(1.00 \pm 0.28[n=30]$ for naïves, $1.01 \pm 0.31$ $[n=25]$ for trained, and $1.03 \pm 0.22[n=24]$ for pseudo-trained). However, as shown in Figure 2A, the level of phosphorylated ERK significantly increased after learning compared with naïve, but not with pseudo-trained (averaged values of pERK/ERK were $1.02 \pm 0.18[n=27]$ for naïves, $1.27 \pm 0.40[n=28]$ for trained, and $1.09 \pm 0.34[n=25]$ for pseudo-trained) (Fig. 2B). The level of activated ERK in the pseudo-trained group had an intermediate averaged value between trained and the naïve groups, and was not significantly different from either $(P=0.33$ for pseudo vs. naïves and $P=0.09$ for pseudo vs. trained). Comparison of the
A

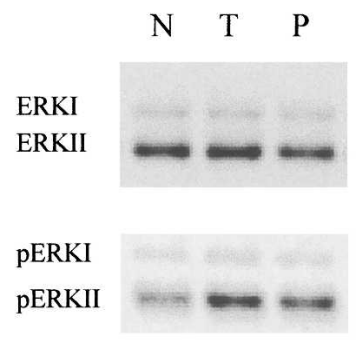

B

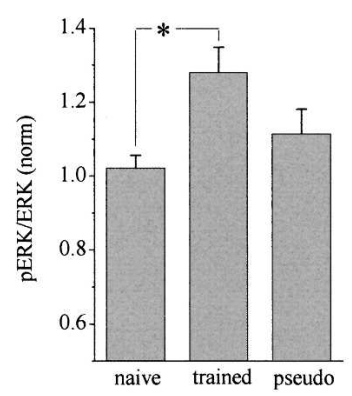

$\mathrm{C}$

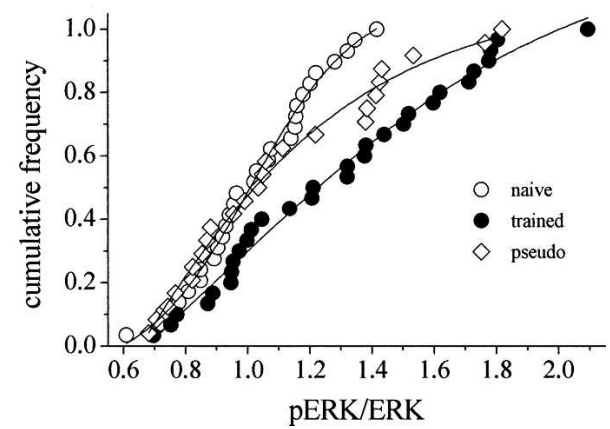

Figure 2. Synaptosomal ERK is activated $3 \mathrm{~d}$ following learning. ( $A$ ) Representative immunoblots for ERKII and phosphorylated ERKII prepared from piriform cortex synaptoneurosomes of each of the three groups (N, naïve; $\mathrm{P}$, pseudo-trained; $\mathrm{T}$, trained). (B) Protein expression level of ERKII is enhanced after olfactory learning. The protein level is normalized to the average value obtained from the naïve animals. Data was taken from 27 naïve, 29 trained, and 25 pseudo-trained rats. The trained group has a significantly higher level of protein expression compared with the naïve group $(P<0.05)$ Summarized data are presented as mean O.D. \pm SE. (C) Cumulative frequency distribution of normalized values from the three groups. Here, each data point represents a rat, and thus it is possible to see whether most data points in one group are modified by treatment. The curve of activated ERK level values in trained rats is smoothly shifted to the left, compared with the naïve group, indicating that such reduction occurred in most of the sampled neurons and not in a subgroup. Two-thirds of the data points of the pseudo-trained group overlap with the naïve group, and the remaining third fits more with the trained group. 
A

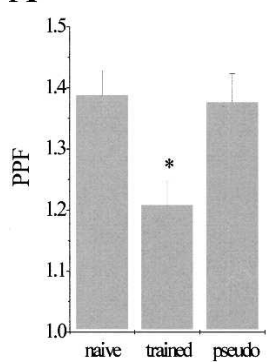

B

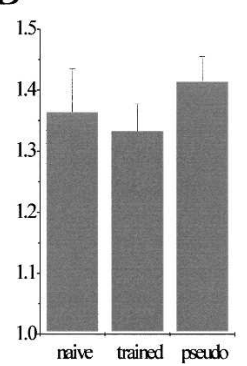

$\mathrm{C}$

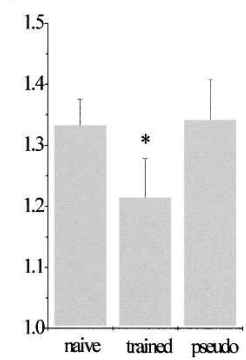

Figure 3. Learning-induced PPF reduction is dependent on PKC activation. $(A)$ PPF is reduced after learning. Control values are obtained from neurons taken from the same slices that were then exposed to the PKC blocker or activator. (B) Application of the PKC inhibitor, GF-109203X, enhanced PPF in the trained group only, as a result of which the difference between the trained and the two control groups was abolished. (C) Application of the PKC activator, OAG, did not affect significantly the PPF value in any of the groups. PPF in NSR was measured in 21 neurons from nine naïve rats, 17 neurons from eight trained rats, and 21 neurons from eight pseudo-trained rats. PPF in GF-109203X was measured in seven neurons from five naïve rats, nine neurons from five trained rats, and six neurons from six pseudo-trained rats. PPF in OAG was measured in nine neurons from seven naïve rats, 10 neurons from five trained rats, and nine neurons from four pseudo-trained rats. Values represent mean $\pm \mathrm{SE}$.

cumulative frequency histograms for the three groups shows that about one-third of the rats in the pseudo-trained group had an increased level of ERK activation, similar to that of most rats in the trained group, while the other two-thirds had values that parallel the values of the naïve group.

The learning-induced increased ERK phosphorylation was specific to the synaptoneurosomes. As previously shown (CohenMatsliah et al. 2007), no differences were observed between groups for the total homogenate fraction (total amount of ERK: naïve $1.00 \pm 0.13, n=27$, pseudo-trained $0.90 \pm 0.23, n=25$, trained $0.92 \pm 0.21, n=29 ; \mathrm{pERK} / \mathrm{ERK}$ : naïve $1.01 \pm 0.19$, $n=27$, pseudo-trained $1.06 \pm 0.26, n=25$, trained $1.05 \pm 0.23$, $n=29$ ). These results suggest that ERK activation is enhanced at the time when learning-relevant PPF becomes apparent; however, enhanced ERK activation is only partially due to rule learning per se. The cumulative frequency of ERKII activation in piriform cortices of rats is shown in Figure 2C. The histogram describing the trained rats is clearly shifted rightward compared with the naïve group, indicating that the learning-induced increased activation of ERK is apparent in most of the brains examined (each data point represents a rat). However, the histogram of the pseudotrained group lies between these two histograms, with two-thirds of the points overlapping with the naïve group, and the remaining third resembling more the trained group. Comparison of cumulative frequency histograms for the three groups favors the possibility that exposure to the odors and/or to the olfactory maze by itself acts to somewhat enhance the level of ERK activation.

\section{PKC activation is necessary but not sufficient} for long-lasting learning-induced PPF reduction

We have previously shown that long-lasting learning-induced enhancement of intrinsic neuronal excitability is dependent on persistent activation of both ERK (Cohen-Matsliah et al. 2007) and PKC (Seroussi et al. 2002). The effect of PKC on neuronal excitability is abolished by blocking MEK, indicating that PKC is upstream relative to the ERK pathway in the sequence of kinase cascade, underlying long-lasting neuronal excitability (CohenMatsliah et al. 2007). Here we examined whether PKC is required for long-term maintenance of PPF reduction.

The PKC blocker, GF-109203X, increased PPF significantly $(P<0.05)$ in neurons from trained rats (from $1.21 \pm 0.16, n=17$ in control solution to $1.33 \pm 0.13, n=9$ in GF-109203X). In sharp contrast, it had no effect on PPF in neurons from naïve (from $1.39 \pm 0.16, n=21$ in control solution to $1.36 \pm 0.18, n=7$ in GF-109203X) and pseudo-trained rats (from $1.38 \pm 0.16, n=21$ in control solution to $1.41 \pm 0.10, n=6$ in GF-109203X). Consequently, the difference between groups was abolished in the presence of the PKC inhibitor (Fig. 3A,B).

The PKC activator OAG had no effect on PPF in any of the groups; in its presence, the averaged values of the naïve $(1.33 \pm 0.13, n=9)$, trained $(1.21 \pm 0.20, n=10)$, and pseudotrained rats $(1.34 \pm 0.20, n=9)$ did not differ from their control values (Fig. 3C).

That OAG by itself does not reduce PPF in neurons from control rats raises the question as to whether PKC is acting upstream or in parallel with ERK. We thus examined whether OAG enhanced ERK activation. No differences were found in the pERKII/ERKII ratio between slices incubated in NSR and OAG (NSR: $1.03 \pm 0.04$; OAG: $0.96 \pm 0.07, n=6$ rats in each group). As discussed below, these results support the notion that in synaptoneurosomes of the piriform cortex, PKC and ERK act in parallel.

\section{Dynamics of learning-induced ERK phosphorylation}

Temporal analysis of learning-induced modulation of synaptic transmission shows that PPF reduction does not appear at the time when rule learning is obtained.

Such PPF reduction occurs only on the third day after completion of training for the second pair of odors, e.g., $4 \mathrm{~d}$ after rule learning (Saar et al. 1999). This reduced value is then maintained for $5 \mathrm{~d}$ (Saar et al. 1999). Such a delay between rule learning and the occurrence of synaptic modification was found not only for PPF reduction, but also for enhanced PSP rise time (Saar et al. 2002) and increased number of spines (Knafo et al. 2005). Thus, the time after rule learning can be divided into two time periods; the period before synaptic modifications, including PPF reduction, is apparent (initial period), and the period after which these modifications are detected (second period).

Here we tested the dynamics of learning-induced synaptic ERK activation during these two time periods. We found that ERK activation levels are enhanced at both periods. Thus, the level of phosphorylated ERK in synaptoneurosomes is elevated prior to PPF reduction, and remains at this high level for the next several days (Fig. 4).

\section{Discussion}

Previous studies have shown that olfactory learning-induced long-lasting enhancement of neuronal excitability is dependent on long-term persistent activation of a second messenger cascade that includes ERK and PKC (Cohen-Matsliah et al. 2007). Here we show that these two key enzymes have also a pivotal role in maintaining learning-induced long-lasting enhancement of synaptic connectivity, as reflected by PPF reduction measured $3 \mathrm{~d}$ after learning. However, as discussed below, here they may act in parallel rather than within the same cascade.

\section{ERK activation modifies synaptic transmission only after learning}

Our data show that PPF in the axons interconnecting piriform cortex layer II pyramidal neurons are subjected to considerable modifications by ERK only after learning. PPF in the pseudotrained rats is also affected to a minor extent, about one-third of that observed in trained neurons. Thus, while most of ERK activation can be attributed to the olfactory-discrimination learning 


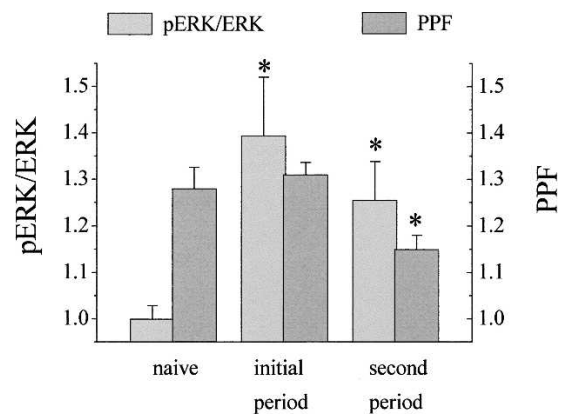

Figure 4. ERK activation occurs prior to PPF reduction. The ratio of pERK to ERK is compared with the averaged value of PPF for three time periods: before training, the first period after rule learning (before PPF reduction occurs), and the second period (after occurrence of PPF reduction). Learning-induced increase in ERK activation is evident prior to PPF reduction. Data for the time course of PPF reduction was taken from Saar et al. (1999). For each averaged value, * notes a significant $(P<0.05)$ difference from the naive group. Averaged PPF was obtained from 23 neurons from naïves, 21 neurons for the initial period, and 19 neurons for the second period. Averaged pERK/ERK was obtained from 29 trained rats, seven rats for the initial period, and 22 rats for the second period. Values represent mean $\pm \mathrm{SE}$.

per se, some of it may be the result of exposing the rats to the maze and/or odors.

We also show that such modifications in synaptic transmission, which last for days after rule learning, are strongly correlated with long-term ERK activation at the synapses. Here too, there is a mild increase in the activity level in the pseudo-trained group. As previously shown (Cohen-Matsliah et al. 2007), the activity level of ERK in the total homogenate fraction, which is known to mediate many cellular events, is not enhanced after learning. This compartment-specific ERK activation, next to the cell membrane, supports the notion of its possible modulation of learning-induced synaptic transmission.

Interestingly, prior to OD learning, ERK has no significant effect on PPF in the piriform cortex, as previously shown in the hippocampus (Rosenblum et al. 2002), although ERK is activated to a considerable level in control conditions. This finding may imply that ERK activation is necessary, but not sufficient for persistent PPF reduction.

\section{PKC activation is required for maintenance of PPF reduction}

We have previously shown that the maintenance of the olfactory learning-induced reduction of the AHP is dependent on PKC (Seroussi et al. 2002), and that the effect of PKC on learninginduced neuronal excitability is mediated by ERK activation (Cohen-Matsliah et al. 2007). A similar sequence of events was suggested to describe the effect of $\mathrm{PKC}$ on the $\mathrm{I}_{\mathrm{A}}$ potassium current (Hu et al. 2003). We thus examined here whether PKC is also instrumental in maintaining learning-induced PPF reduction, at the same time when ERK appears to have such a role.

We found that blocking PKC activation enhances PPF in neurons from trained rats to the level observed in the two control groups. Thus, persistent PKC activation is necessary for maintaining such long-lasting PPF reduction. However, PKC activation by itself does not induce PPF reduction; application of its activator, OAG, did not affect PPF in any of the groups, indicating that an additional process is required. Thus, it may be possible that ERK and PKC are activated in parallel in an AND gate, which is necessary for maintaining long-lasting PPF reduction. That OAG application does not enhance ERK activation in brain slices, lends further support to this conclusion.
Time-specific effects of ERK on learning and memory

Direct evidence in support of a role for ERK in memory has been accumulating rapidly over the past decade. Although the evidence stems from findings involving different learning types and brain areas, they all show a pattern of temporal ERK activation, initiated minutes to several hours after training completion (Atkins et al. 1998; Berman et al. 1998; Blum et al. 1999; Schafe et al. 2000; Belelovsky et al. 2005; Herry et al. 2006). The picture emerging from these previous studies is that at relatively early stages of memory formation and consolidation, ERK modulates learning-relevant processes by affecting different substrates, which are yet to be identified.

Our study shows that for days after learning completion, ERK activation is still significantly enhanced at the synapses. Interestingly, such ERK activation occurs $2 \mathrm{~d}$ before PPF reduction occurs. This result suggests that enhanced and prolonged ERK activation is involved in the induction of the process leading to PPF reduction, rather than only in its maintenance.

How is ERK activation maintained for days? Previous work suggests that persistent activation of the kinases in the ERK cascade may form a positive feedback loop that could counteract the loss of ERK activity due to the protein turnover and signal withdrawal. Such a system has the potential to store information. However, such activation is restricted to small volumes (Bhalla and Lyengar 1999; Smolen et al. 2008). Since MEK and ERK are known to localize in synaptoneurosomes (Suzuki et al. 1995), and we have shown activation of ERK lasting days after learning in those cellular compartments, our results lend support to this hypothesis.

How does ERK affect learning-induced presynaptic transmission? One intriguing possibility is pointed out by a study showing that ERK activation affects synaptic release by phosphorylation of synapsin I, which is instrumental in learning-related enhanced synaptic release (Kushner et al. 2005). Whether a similar mechanism underlies such long-lasting enhanced synaptic transmission after OD rule learning remains to be explored.

In conclusion, our study shows that ERK can be activated locally at synaptoneurosomes, many days following learning. Moreover, this induced ERK activation modulates synaptic function. This ERK activation is crucial for maintaining learninginduced PPF reduction in the first days after learning completion.

\section{Materials and Methods}

\section{Animal training}

\section{Subjects and apparatus}

Age-matched young adult Sprague-Dawly male rats were used. Prior to training they were maintained on a $23.5 \mathrm{~h}$ waterdeprivation schedule, with food available ad libitum. Olfactory discrimination training protocol was performed daily on each trained and pseudo-trained rat in a 4-arm radial maze, as previously described (Saar et al. 1999, 2001), with commercial odors that are regularly used in the cosmetics and food industry.

\section{Training}

Olfactory training consisted of 20 trials per day for each rat as previously described (Saar et al. 2001). In short, in each trial the rat had to choose between two odors (positive and negative cue) presented simultaneously. Rats designated to the trained group were rewarded upon choosing the positive cue. Rats in the pseudo-trained group were rewarded in a random fashion, upon choosing any odor. The criterion for learning was at least eight positive-cue choices in the last 10 trials of a training day, as was previously used (Staubli et al.1987; Saar et al.1999, 2001). Rats in the naïve group were water restricted, but not exposed to the maze. Typically, two to three trained rats and two to three pseudotrained rats were trained at the same training period, and all of the 
rats in the trained group had to meet the criteria for the first pair of odors before all trained and pseudo-trained rats were exposed to a second pair of odors. Training for a new pair began only after training for the second pair was completed for all rats. As previously described (Saar et al. 1999, 2001), rats indeed learned the second and third pairs of odors much faster than the first pair (7-8 $\mathrm{d}$ of training for the first pair and 1-2 $\mathrm{d}$ for the second and third pairs). Several rats were trained with a second and third pair of odors. Such training was always completed within $1 \mathrm{~d}$. Thus, rule learning was considered to occur on the day when rats completed training for the first pair of odors.

\section{Slice preparation, stimulation, and recording}

A total of $400-\mu \mathrm{m}$ coronal brain slices were cut as previously described (Saar et al. 1998) and kept in oxygenated $\left(95 \% \mathrm{O}_{2}+5 \%\right.$ $\mathrm{CO}_{2}$ ) normal saline Ringers' (NSR) solution (in millimolar: $\mathrm{NaCl}$ $124, \mathrm{KCl} 3, \mathrm{MgSO}_{4} 2, \mathrm{NaH}_{2} \mathrm{PO}_{4} 1.25, \mathrm{NaHCO}_{3} 26, \mathrm{CaCl}_{2} 2$, and glucose 10). Intracellular recordings were obtained from pyramidal cells in layer II of the piriform cortex, with $4 \mathrm{M}$ K-acetatefilled sharp glass microelectrodes, at $35^{\circ} \mathrm{C}$. Several piriform cortex slices were obtained from each rat.

PPF were recorded within minutes after good recording conditions were established (resting potential of at least $-65 \mathrm{mV}$ and action potential amplitude of $80 \mathrm{mV}$ or more). Tungsten electrodes were placed in layer $\mathrm{Ib}$ of the piriform cortex to stimulate the intrinsic fibers. Electrical stimuli were applied at $0.1 \mathrm{~Hz}$. The amplitudes of the responses were measured from digital averaging of 10 consecutive responses. To standardize the intracellular recording conditions, stimulus intensity was adjusted so that the averaged amplitude of 10 consecutive post-synaptic potentials (PSPs) in the recorded cell would be $10 \mathrm{mV}$ at $\mathrm{Vm}=-80 \mathrm{mV}$. After drug application, the stimulus intensity was adjusted again to evoke a first PSP with amplitude of $10 \mathrm{mV}$.

The identity of the rat from which neurons were recorded (naïve, trained, or pseudo-trained) was not known to the person conducting the experiments and measurements.

\section{Drug application}

All drugs were applied into the perfusing Ringer solution at the following concentrations: MEK inhibitor PD98059 (Sigma), 38 $\mu \mathrm{M}$; PKC activator 1-oleoyl-20acety-sn-glycerol (OAG) (RBI), 10 $\mu \mathrm{M}$; PKC inhibitor bisindoylmaleimide (GF-109203X), (Sigma), $10 \mu \mathrm{M}$. Slices were exposed to drugs for at least $20 \mathrm{~min}$ before the recordings. In each recording condition, several cells were recorded before and after drug application, while others were recorded under one condition only (e.g., in control solution or in the presence of the drug).

\section{Sample preparation}

\section{Preparation of synaptoneurosomes}

Preparation of synaptoneurosomes of piriform cortex was obtained as described (Quinlan et al. 1999; Belelovsky et al. 2005). Briefly, tissues were homogenized in $4 \mathrm{~mL}$ of homogenization buffer (in millimolar: HEPES 10, EDTA 2, EGTA 2, DTT 0.5, 1\% phosphatase inhibitor cocktail [Sigma] and 1\% protease inhibitor cocktail [Sigma]). Eighty microliters of the homogenized tissue were removed for analysis of total protein fractions. The remaining homogenate was then filtered subsequently through a 100$\mu \mathrm{m}$ filter, followed by a 5 - $\mu \mathrm{m}$ filter (Milliopre) and centrifuged at $1000 \mathrm{~g}$ for $10 \mathrm{~min}$ at $4^{\circ} \mathrm{C}$. The pellet containing the synaptoneurosomes was resuspended in homogenizing buffer and subject to SDS-PAGE following protein determination.

\section{Western blot analysis}

All samples were resolved by SDS-PAGE and transferred to nitrocellulose membrane (BioRad Laboratories, Inc.) for immunoblotting. Blots were visualized with enhanced chemiluminescence (ECL Plus, Amersham Biosciences) and quantified using a CCD camera (XRS, BioRad Laboratories, Inc.) and "Quantity-One" software. Here, we quantified ERKII (thus, in the Results section, ERK is intended to note ERKII). The ratio between phosphory- lated ERK and the total amount of the protein for each rat was calculated and normalized to the naïve group average run on the same gel.

To measure the effect of PKC activation on ERK phosphorylation in synaptoneurosomes, piriform cortices were isolated from $400-\mu \mathrm{m}$ coronal brain slices. Slices were incubated in normal slice Ringer (NSR) for $60 \mathrm{~min}$, followed by additional incubation of $40 \mathrm{~min}$ in NSR or OAG $(10 \mu \mathrm{M})$. Subsequently, synaptoneurosomes were prepared from the slices, as described above.

\section{Reagents}

p44/42 MAP kinase antibody (1:1000), rabbit polyclonal; phospho-p44/42 MAP kinase (Thr202/Tyr204) antibody (1:500) were purchased from Cell Signaling. Goat anti-rabbit IgG was obtained from Jackson ImmunoResearch Laboratories, Inc. and used at a concentration of 1:20,000.

\section{Statistical analysis}

For all of the electrophysiological measurements, betweengroups comparison was done using one-way ANOVA for the three groups (naïve, trained, and pseudo-trained), and post-hoc multiple $t$-tests were then applied to compare between each of the two groups. The effect of the drugs on each of the three groups was evaluated by comparing the recordings from the same group, with and without the treatment, using independent $t$ tests. Values throughout the text are presented as mean \pm SD. Data in graphs are presented as mean $\pm \mathrm{SE}$.

\section{Acknowledgments}

This work was supported by grants from the United States-Israel Binational Science Foundation and the Israel Science Foundation.

\section{References}

Adams, J.P. and Sweatt, J.D. 2002. Molecular psychology: Roles for the ERK MAP kinase cascade in memory. Annu. Rev. Pharmacol. Toxicol. 42: $135-163$.

Atkins, C.M., Selcher, J.C., Petraitis, J.J., Trzaskos, J.M., and Sweatt, J.D. 1998. The MAPK cascade is required for mammalian associative learning. Nat. Neurosci. 1: 602-609.

Belelovsky, K., Elkobi, A., Kaphzan, H., Nairn, A.C., and Rosenblum, K. 2005. A molecular switch for translational control in taste memory consolidation. Eur. J. Neurosci. 22: 2560-2568.

Berman, D.E., Hazvi, S., Rosenblum, K., Seger, R., and Dudai, Y. 1998. Specific and differential activation of mitogen-activated protein kinase cascades by unfamiliar taste in the insular cortex of the behaving rat. J. Neurosci. 18: $10037-10044$.

Bhalla, U.S. and Lyengar, R. 1999. Emergent properties of networks of biological signaling pathways. Science 283: 381-386.

Blum, S., Moore, A.N., Adams, F., and Dash, P.K. 1999. A mitogen-activated protein kinase cascade in the CA1/CA2 subfield of the dorsal hippocampus is essential for long-term spatial memory. $J$. Neurosci. 19: 3535-3544.

Brosh, I., Rosenblum, K., and Barkai, E. 2007. Learning-induced modulation of SK channels-mediated effect on synaptic transmission. Eur. J. Neurosci. 26: 3253-3260.

Cohen-Matsliah, S.I., Brosh, I., Rosenblum, K., and Barkai, E. 2007. A novel role for extracellular signal-regulated kinase in maintaining long-term memory-relevant excitability changes. J. Neurosci. 27: $12584-12589$.

Crow, T., Xue-Bian, J.J., Siddiqi, V., Kang, Y., and Neary, J.T. 1998. Phosphorylation of mitogen-activated protein kinase by one-trial and multi-trial classical conditioning. J. Neurosci. 18: 3480-3487.

Di Cristo, G., Berardi, N., Putignano, E., Ratto, G.M., and Maffei, L. 2001. Requirement of ERK activation for visual cortical plasticity. Science 292: 2337-2340.

English, J.D. and Sweatt, J.D. 1996. Activation of p42 mitogen-activated protein kinase in hippocampal long term potentiation. J. Biol. Chem. 271: 24329-24332.

English, J.D. and Sweatt, J.D. 1997. A requirement for the mitogen-activated protein kinase cascade in hippocampal long-term potentiation. J. Biol. Chem. 272: 19103-19106.

Herry, C., Trifilieff, P., Micheau, J., Luthi, A., and Mons, N. 2006 Extinction of auditory fear conditioning requires MAPK/ERK activation in the basolateral amygdala. Eur. J. Neurosci. 24: 261-269.

$\mathrm{Hu}$, H.J., Glauner, K.S., and Gereau IV, R.W. 2003. ERK integrates PKA and PKC signaling in superficial dorsal horn neurons. I. Modulation 
of A-type $\mathrm{K}^{+}$currents. J. Neurophysiol. 90: 1671-1679.

Knafo, S., Libersat, F., and Barkai, E. 2005. Olfactory learning-induced morphological modifications in single dendritic spines of young rats. Eur. J. Neurosci. 21: 2217-2226.

Kushner, S.A., Elgersma, Y., Murphy, G.G., Jaarsma, D., van Woerden, G.M., Hojjati, M.R., Cui, Y., LeBoutillier, J.C., Marrone, D.F., Choi, E.S., et al. 2005. Modulation of presynaptic plasticity and learning by the H-ras/extracellular signal-regulated kinase/synapsin I signaling pathway. J. Neurosci. 25: 9721-9734.

Quinlan, E.M., Philpot, B.D., Huganir, R.L., and Bear, M.F. 1999. Rapid, experience-dependent expression of synaptic NMDA receptors in visual cortex in vivo. Nat. Neurosci. 2: 352-357.

Rosenblum, K., Futter, M., Jones, M., Hulme, E.C., and Bliss, T.V. 2000. ERKI/II regulation by the muscarinic acetylcholine receptors in neurons. J. Neurosci. 20: 977-985.

Rosenblum, K., Futter, M., Voss, K., Erent, M., Skehel, P.A., French, P., Obosi, L., Jones, M.W., and Bliss, T.V. 2002. The role of extracellular regulated kinases I/II in late-phase long-term potentiation. $J$. Neurosci. 22: 5432-5441.

Saar, D., Grossman, Y., and Barkai, E. 1998. Reduced after-hyperpolarization in rat piriform cortex pyramidal neurons is associated with increased learning capability during operant conditioning. Eur. J. Neurosci. 10: 1518-1523.

Saar, D., Grossman, Y., and Barkai, E. 1999. Reduced synaptic facilitation between pyramidal neurons in the piriform cortex after odor learning. J. Neurosci. 19: 8616-8622.

Saar, D., Grossman, Y., and Barkai, E. 2001. Long-lasting cholinergic modulation underlies rule learning in rats. J. Neurosci. 21: 1385-1392.
Saar, D., Grossman, Y., and Barkai, E. 2002. Learning-induced enhancement of postsynaptic potentials in pyramidal neurons. $J$. Neurophysiol. 87: 2358-2363.

Schafe, G.E., Atkins, C.M., Swank, M.W., Bauer, E.P., Sweatt, J.D., and LeDoux, J.E. 2000. Activation of ERK/MAP kinase in the amygdala is required for memory consolidation of Pavlovian fear conditioning. $J$. Neurosci. 20: 8177-8187.

Selcher, J.C., Weeber, E.J., Christian, J., Nekrasova, T., Landreth, G.E., and Sweatt, J.D. 2003. A role for ERK MAP kinase in physiologic temporal integration in hippocampal area CA1. Learn. Mem. 10: $26-39$

Seroussi, Y., Brosh, I., and Barkai, E. 2002. Learning-induced reduction in post-burst after-hyperpolarization (AHP) is mediated by activation of PKC. Eur. J. Neurosci. 16: 965-969.

Smolen, P., Douglas, A., and Byrne, J.H. 2008. Bistable MAP kinase activity: A plausible mechanism contributing to maintenance of late long-term potentiation. Am. J. Physiol. 294: C503-C515.

Staubli, U., Fraser, D., Faraday, R., and Lynch, G. 1987. Olfaction and the "data" memory system in rats. Behav. Neurosci. 101: 757-765.

Suzuki, T., Okumura-Noji, K., and Nishida, E. 1995. ERK2-type mitogen-activated protein kinase and its substrates in post synaptic density fractions from the rat brain. Neurosci. Res. 22: 277-285.

Sweatt, J.D. 2004. Mitogen-activated protein kinases in synaptic plasticity and memory. Curr. Opin. Neurobiol. 14: 311-317.

Thomas, G.M. and Huganir, R.L. 2004. MAPK cascade signalling and synaptic plasticity. Nat. Rev. Neurosci. 5: 173-183.

Received June 30, 2008; accepted in revised form July 17, 2008. 


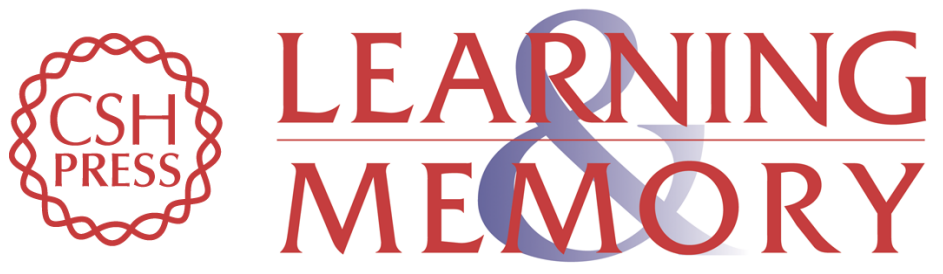

\section{Persistent ERK activation maintains learning-induced long-lasting modulation of synaptic connectivity}

Sivan Ida Cohen-Matsliah, Yaron Seroussi, Kobi Rosenblum, et al.

Learn. Mem. 2008, 15:

Access the most recent version at doi:10.1101//m.1127008

References This article cites 31 articles, 15 of which can be accessed free at:

http://learnmem.cshlp.org/content/15/10/756.full.html\#ref-list-1

License

Email Alerting Receive free email alerts when new articles cite this article - sign up in the box at the Service top right corner of the article or click here. 